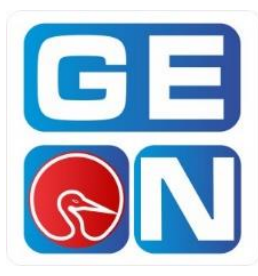

Revista GEON (Gestión, Organizaciones y Negocios). ISSN: 2346-3910 en línea revistageon@unillanos.edu.co Universidad de los Llanos Colombia

Ramírez Tapias, Diego Alejandro ${ }^{i}$.

La responsabilidad social de los Hospitales del Estado frente a las teorías de la gerencia del valor. Caso aplicado ESE Hospital Venancio Díaz Díaz. Revista GEON, Vol. 4, No. 1, 2017

Pág. 91-102

Disponible en: $\underline{\text { https://doi.org/10.22579/23463910.47 }}$

${ }^{\mathrm{i}}$ https://orcid.org/0000-0002-3609-3320

Esta publicación se encuentra bajo licencia: Creative Commons

ReconocimientoNoComercialSinObraDerivada 4.0 Internacional

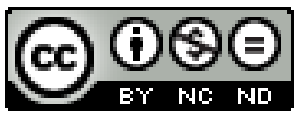

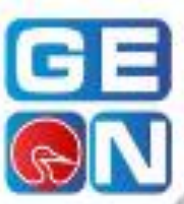

66

Los Estados Financieros de la ESE Hospital Venancio Díaz Díaz muestra un claro y continuo deterioro en la generación de valor, ocasionado. principalmente por la ineficiencia operativa

\section{RevistaGEON} (in) 9 :
Ramirez Topias, 0. [20V]. La Responsobilidod Social de los Hospitales del Estado Frente a las Teorias de la Gerencia del Volor. Coso Aplicado ESE Hospital Venancio Diaz Diaz 


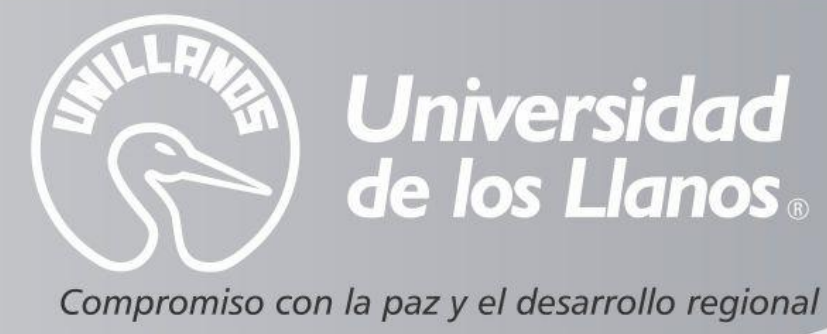

\section{REVISTA}

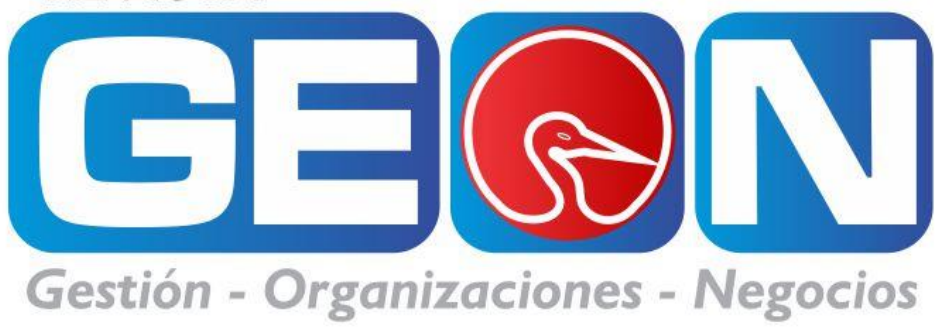

ISSN 2346 - 3910 (En línea)

Voltumen 4No 0 enero - ffunfo 2007

Revista GEON Facultad de Ciencias Económicas de la Universidad de los Llanos

VILLAVICENCIO - COLOMBIA

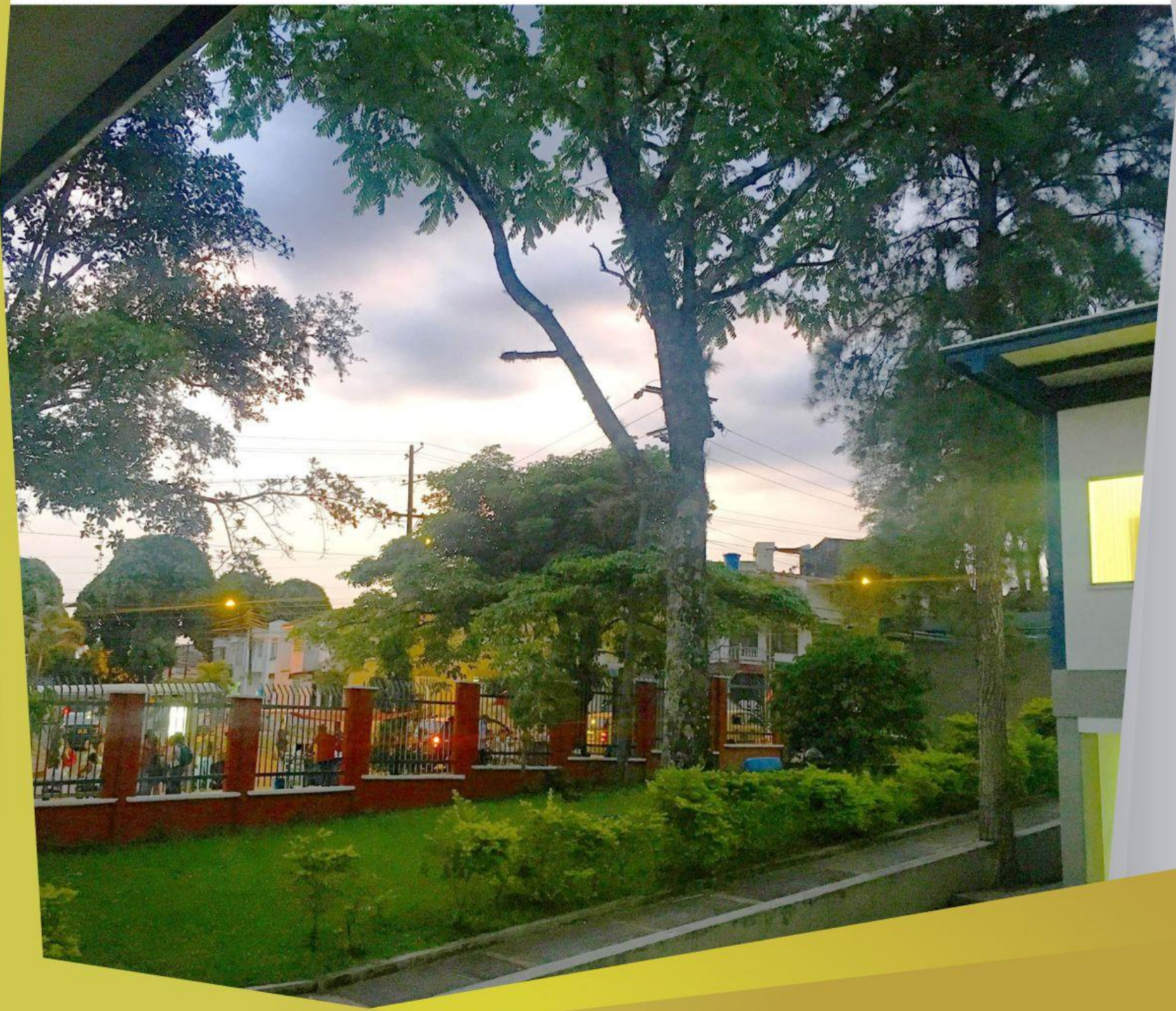




\title{
La Responsabilidad Social de los Hospitales del Estado Frente a las Teorías de la Gerencia del Valor. Caso Aplicado ESE Hospital Venancio Díaz Díaz por Diego Alejandro Ramírez Tapias
}

\author{
The Social Responsibility of the State Hospitals Faced with the Theories of the Value Management. \\ Case Applied ESE Hospital Venancio Díaz Díaz by Diego Alejandro Ramírez Tapias
}

Diego Alejandro Ramírez Tapias

Contador Público

Articulo recibido 2016/08/30

Corporación Universitaria de Sabaneta

aceptado 2016/11/24

Diego.ramirez@unisabaneta.edu.co

\section{Resumen}

Como momento inicial el trabajo resume la actual situación de la salud en Colombia para luego continuar evaluando los resultados de la gestión de la ESE Hospital Venancio Díaz Díaz con las teorías tradicionales y la gerencia del valor. Específicamente el análisis se basa en el cálculo de indicadores tradicionales y se centra en el análisis del EVA de la entidad para los años 2012-2015. Después de obtener los resultados financieros, el escrito resuelve el dilema de si este tipo de empresas debe siempre buscar la rentabilidad económica, o si por el contrario, un impacto social positivo los libera de ser sostenibles cuando los dos objetivos no pueden alcanzarse (Rentabilidad e impacto social). El artículo en su parte final se complementa con una evaluación de la responsabilidad social de este hospital en el municipio donde ejerce influencia.

Palabras Claves: hospitales, salud, Valor agregado

\begin{abstract}
As a starting point, the paper summarizes the current health situation in Colombia and then continues to evaluate the results of the management of the Venancio Diaz Diaz Hospital with traditional theories and value management. Specifically, the analysis is based on the calculation of traditional indicators and focuses on the analysis of the entity's EVA for the years 2012-2015. After obtaining the financial results, the paper solves the dilemma of whether these types of companies should always seek economic profitability, or if, on the contrary, a positive social impact frees them from being sustainable when both objectives can not be achieved (Profitability and Social impact). The article in its final part is complemented with an evaluation of the social responsibility of this hospital in the municipality where it exerts influence.
\end{abstract}

Key words: hospitals, health, Value added 


\section{Introducción}

Los recursos públicos por ser activos de la sociedad deben constituirse en objeto de especial cuidado por parte de las personas que los manejan, y es la misma sociedad quien se debe encargar de asegurar la inversión necesaria para mantener el nivel de vida de sus ciudadanos. Según como lo establece INTOSAI citado por (Moreno, 2006).

"El Control no representa una finalidad en sí mismo, sino una parte imprescindible de un mecanismo regulador que debe señalar oportunamente las desviaciones normativas y las infracciones de los principios de legalidad, rentabilidad, utilidad y racionalidad de las operaciones financieras, de tal modo que puedan adoptarse las medidas correctivas convenientes en cada caso, determinarse la responsabilidad del órgano culpable, exigirse la indemnización correspondiente o adoptarse las determinaciones que impidan o por lo menos, dificulten la repetición de tales infracciones en el futuro".

Es el control entonces la herramienta utilizada por la sociedad para ejercer un derecho a exigir el correcto uso de los recursos bajo parámetros de legalidad, rentabilidad, utilidad y racionalidad, siempre actuando bajo principios financieros. De no alcanzarse los objetivos propuestos, la sociedad puede exigir cualquier reparación que contravenga los daños ocasionados.

Pero, ¿cuál es el estado en el control de los recursos en Colombia? La conclusión se evidencia en el siguiente punto de vista:

"Aunque hay que reconocer los importantes avances tendido y mejoras en el marco formal del control fiscal, como el desmonte del proceso de coadministración, desafortunadamente al día de hoy no han logrado obtener un nivel eficaz del sistema, ya que durante su ejecución han surgido diversas dificultades no esperadas, debido a que en muchas oportunidades la forma como vienen practicando el control fiscal posterior, tiende a llegar de forma extemporánea, cuando los detrimentos son un hecho ya consumado, lo que no ayuda a combatir el alto grado de corrupción y manejo cuestionable de los fondos públicos; por lo hay que alertar no solo a las autoridades, sino también a la comunidad, quien al final es la más afectada por la deficiencia del sistema" (Vergara, 2013).

El autor es claro en afirmar que el control de los recursos públicos en Colombia ha avanzado, pero requiere de herramientas previas a la ocurrencia de los hechos. La misma normatividad le ha concedido a la sociedad la potestad de aplicar mecanismos para la detección de fraudes (veedurías ciudadanas, control social) siempre en pro de evitar pérdidas importantes de recursos que pueden servir para ofrecer bienes públicos de calidad.

\section{Los Recursos Públicos y la Salud en Colombia}

Según el anterior panorama, el control de los recursos en Colombia requiere de mejoras para anticiparse a situaciones que pueden ocasionar pérdidas considerables. Bajo esta óptica, la salud, por ser un derecho fundamental del ciudadano debe incluirse dentro de la aplicación de las teorías de control.

En nuestro país el sistema de salud atraviesa una grave crisis caracterizada por la falta de vigilancia operativa y el lucro de los operadores de salud (EPS). A pesar de alcanzar una cobertura casi universal, la calidad de los servicios ha disminuido hasta el punto de condicionarla a la capacidad de pago del usuario. Lo anterior, sumado a las crisis financieras por desvió de recursos han convertido el sistema en el causante de graves ineficiencias operativas evidenciadas en la baja calidad del servicio (Triviño, 2013) 
Basado en lo anterior es claro concluir que los recursos públicos dispuestos para el sector salud no han impactado positivamente en la población ocasionando que la sociedad pierda oportunidades para recibir atención de calidad. La universalidad y calidad en la atención se ha visto reducida a causa de sonados desfalcos en el sistema.

En vista de la crisis descrita se genera un interrogante relacionado con la rentabilidad que debe perseguir una entidad pública, que, si bien su finalidad no es el lucro económico, debe responder a estándares financieros para asegurar su operación. En el artículo 4 literal c se establece como objetivo de las ESE asegurar a través de un manejo administrativo adecuado, la rentabilidad social y financiera de la Empresa Social. (Minsalud, 1994).

La misma norma establece que la red pública hospitalaria debe alcanzar por si misma su sostenibilidad procurando mantener la calidad de sus servicios para ofrecer a la ciudadanía ejercer su derecho constitucional a la salud. Pero ante la crisis queda el interrogante de cómo la continuidad de las Empresas Sociales del Estado se ve afectada por el detrimento en sus indicadores, lo que conlleva a una entidad del estado a entrar en situación de riesgo fiscal. Como lo plantea el periódico El Universal en su edición del 13 de junio de 2016 (universal, 2016), el 25\% de la red hospitalaria pública se encuentra en riesgo financiero, indicador que establece la proporción de entidades del estado que requieren un saneamiento financiero inmediato. $\mathrm{Y}$ aunque el informe enumera las estadísticas del informe, también plantea los errores en la medición del indicador, dejando por fuera entidades que presentan una crisis más grave de lo que sus informes financieros reflejan. Por último, el sistema de ajuste financiero tiene la bondad de alcanzar una sostenibilidad financiera, pero debido a las exigencias de la norma cuando estos programas son aplicados en los hospitales generan inviabilidad social por los recortes en sus recursos de operación.

\section{Situación particular ESE Venancio Díaz de Sabaneta}

Los centros de atención en salud Empresas Sociales de Estado (Antiguamente conocidos como hospitales) no son ajenos a la crisis del sector, su objeto social se afecta gravemente con la falta de fluidez de recursos para atender su operación. Para (Burgos, Montes, \& García, 2013) los problemas de la red pública hospitalaria obedecen a cambios recurrentes en la normatividad, dificultades en la consecución de recursos para operar, demora en los pagos de los entes pertenecientes al sistema (EPS, FOSYGA) $\mathrm{y}$ altos costos laborales. Lo anterior ocasiona problemas de liquidez asociadas a altos indicadores de cartera vencida.

Uno de los compromisos de la academia es establecer mecanismos para articular el sector real de la economía con las teorías aplicadas en la enseñanza. Para tal fin, y como rango de acción de UNISABANETA, la institución se propone describir la situación financiera de la ESE Hospital Venancio Díaz, la cual, según información preliminar extraída de algunas fuentes secundarias (noticias, casos particulares) presenta graves problemas en su operación, incluso con demoras en el pago de sus obligaciones.

Precisamente, el objeto de la investigación es determinar en un primer momento cual es la realidad de esas hipótesis, contrastando la situación financiera basada en la gestión del valor, con su operación real. En un segundo momento, la investigación analiza la situación de la entidad, aplicando métodos financieros tradicionales y aquellos basados en la gerencia del valor, específicamente, con el cálculo y análisis del EVA para los periodos 2012-2015. La investigación termina revisando información acerca del cumplimiento de la responsabilidad empresarial de ESE hospital Venancio Díaz Díaz. Por último, se concluye si es posible alcanzar los objetivos financieros siendo una entidad del sector salud que cumple con los compromisos sociales que le fueron 
encomendados por normas legales (Responsabilidad social empresarial)

Unas de las bondades de este proyecto es asegurar a la comunidad del municipio de Sabaneta que los recursos administrados por el hospital son invertidos eficientemente y que la entidad puede contribuir al mejoramiento de la salud de los ciudadanos del municipio.

\section{Objetivos}

\section{Objetivo general}

Valorar la RSE de la ESE Hospital Venancio Díaz frente a la sostenibilidad financiera reflejada en el EVA.

\section{Objetivos específicos}

1. Calcular el EVA basado en los Estados Financieros e indicadores de la ESE Hospital Venancio Díaz

2. Evaluar la responsabilidad social empresarial de la ESE hospital Venancio Díaz en el municipio de Sabaneta (Antioquia).

3. Comparar la RSE de la ESE hospital Venancio Díaz con los resultados financieros obtenidos

\section{Contexto teórico}

La ESE Hospital Venancio Díaz es una entidad prestadora de servicios de primer nivel de complejidad y atención especializada de segundo nivel especializada en la promoción de la salud y prevención de la enfermedad. (Díaz, 2004). La entidad enmarca su operación en lo reglamentado por la ley 100 de 1993, la cual establece que la prestación de servicios de salud en forma directa por la Nación o por las entidades territoriales, se realizará principalmente por intermedio de las empresas sociales del Estado, que constituyen una categoría especial de entidad pública descentralizada, con personería jurídica, patrimonio propio y autonomía administrativa, creadas por la ley o por las asambleas o concejos, según el caso. (Congreso de la República, 1993)

Adicionalmente, el objetivo es la prestación de los servicios de salud, como servicio público a cargo del Estado o como parte del servicio público de seguridad social. (Congreso de la República, 1993)

Precisamente para analizar la situación de esta entidad, a la luz de la norma que autoriza su operación, y sobre todo para determinar el uso de los recursos, se emplean herramientas de análisis financiero, las cuales forman parte de un sistema o proceso de información cuya misión es la de aportar datos que permitan conocer la situación financiera actual de la empresa y pronosticar su futuro, lo que resulta de gran interés para la sociedad actual. (Álvarez, 2004)

A su vez, Hernández (2005), define el análisis financiero como una técnica de evaluación del comportamiento operativo de una empresa, que facilita el diagnóstico de la situación actual y la predicción de cualquier acontecimiento futuro. Se orienta hacia la consecución de objetivos preestablecidos.

Este análisis se basa en la interpretación de los hechos financieros acontecidos en el desarrollo de la actividad empresarial, para lo cual utiliza métodos que una vez aplicados llevan a una toma de decisiones acertadas; sumado a que contribuye a examinar la capacidad de endeudamiento e inversión de la empresa, tomando como punto de inicio la información aportada por los estados financieros. (Rosillón, 2009)

Frente a la utilización de los métodos de análisis financiero (Mares, 2009), establece como análisis previo la metodología que consiste en el uso de varias técnicas clásicas como el análisis vertical (porcientos integrales) el análisis horizontal (aumentos y disminuciones), el análisis histórico a través de tendencias y comparaciones, y el análisis proyectado o estimado. Su resultado final es la obtención de un ratio o indicador. Para complementar el análisis se utiliza el juicio personal para emitir una opinión acerca del análisis efectuado. 
En contraposición a los métodos tradicionales, surge otro que persigue un objetivo diferente al tradicional, partiendo del valor como fundamento principal. Para entender el significado financiero de valor, (Colina, 2016) establece que una inversión aporta a la creación de valor cuando retorna un monto de dinero superior a lo invertido inicialmente, y cubre todos los costos asociados, incluyendo los costos de oportunidad de los recursos invertidos.

Luego del análisis de valor desde la óptica financiera, es necesario entender la filosofía para alcanzar el valor como objetivo primordial para maximizar la riqueza de los propietarios. Tal filosofía es la gerencia del valor y (García, 2003) la entiende como el conjunto de procesos administrativos integrados a la dirección estratégica de una empresa que tiene como objetivo principal la toma de decisiones enfocada al aumento del valor

La teoría explica la metodología para alcanzar el valor en los procesos organizacionales. Se crea valor por medio de tres aspectos: Direccionamiento Estratégico, la Gestión Financiera y la Gestión del Talento Humano. (García, 2003).

A través del Direccionamiento Estratégico la creación de valor se fomenta implementando dos procesos: 1) Adopción de Mentalidad Estratégica; 2) Implementación de la Estrategia.

A través de la Gestión Financiera la creación de valor se fomenta implementando cuatro procesos:

1. Definición y Gestión de los Inductores de Valor.

2. Identificación y Gestión de los Micro inductores de Valor.

3. Valoración de la Empresa.

4. Monitoreo del Valor.

Finalmente, a través de la gestión del talento humano la creación de valor se fomenta implementando dos procesos: 1) Educación, Entrenamiento y Comunicación;
Compensación atada a resultados asociados con el valor.

Desde la óptica financiera se señala la dinámica para establecer los criterios necesarios para implementar la gerencia del valor en las organizaciones (García, 2003):

Definir y Gestionar Inductores de Valor. Este proceso consiste en determinar cuál o cuáles son las variables asociadas con la operación de la empresa que más inciden o afectan su valor, para luego promover en todos los niveles de la organización el alineamiento de las decisiones hacia la mejora permanente de esas variables, denominadas Inductores de Valor. Se definirá Inductor de Valor como aquel aspecto de la operación de la empresa que está atado en relación causa-efecto a su valor y por lo tanto permite explicar el porqué de su aumento o disminución como consecuencia de las decisiones tomadas. Los capítulos 3 al 6 abordan el estudio de dichos inductores

Identificar y Gestionar Micro Inductores de Valor. La cuarta categoría de inductores de valor, los Micro inductores se relacionan con aquellos aspectos muy particulares de la empresa cuyo comportamiento afecta los inductores operativos y financieros.

Los micros inductores de valor son indicadores que a la medida de las características de la empresa están asociados con el mejoramiento de los inductores operativos y financieros. Se relacionan con los denominados "Indicadores de Desempeño" o "Indicadores de Gestión”.

Según los referentes anteriores, es claro concluir que una de las finalidades organizacionales es crear valor para sus accionistas a través de decisiones administrativas encaminadas a máxima su riqueza. Pero, desde el aspecto no lucrativo, especialmente, de las entidades estatales encargadas de prestar servicios de salud, debe incorporarse aspectos no cuantitativos, o al menos, considerar otros factores diferentes a los financieros, esto es, el cumplimiento de estándares de responsabilidad social empresarial. 
"Podemos considerar que la responsabilidad es la contraparte de los derechos que una entidad ejerce mediante una voluntad. El contenido de la responsabilidad social de las empresas comprende las acciones intencionales de los empresarios en que se expresa su estrategia global así como los impactos directos e indirectos de tales acciones en la sociedad, como, por ejemplo: la conformación de horarios de vida, la influencia de la tecnología de las empresas sobre hábitos alimenticios, la percepción del ciudadano común sobre el tiempo, el espacio y el sentido del trabajo y, en general, sobre el significado de la vida" (Andrade \& Fernández, 2010).

\section{Ejes de la RSE Para el Sector Salud}

Según (Morales \& Galeano, 2013) la RSE en el sector salud se debe basar en 6 ejes transversales para alcanzar la sostenibilidad en estas entidades, estos elementos se describen a continuación:

1. Gobernanza e Inversión Transparente: La gobernanza es fundamental para el ejercicio de la Responsabilidad Social empresarial, porque desde allí se definen las políticas que rigen el ejercicio de la organización, es todo el sistema por el cual la toma de decisiones de una empresa se realiza con sentido ético y transparente. Esta característica abarca aspectos tales como la comunicación en todos los niveles de la organización niveles de la organización, la definición de cánones de Igualdad, rendición de cuentas y usos eficiente de los recursos financieros asegurando que se haga de forma ética $y$ transparente.

2. Prácticas Laborales Justas y Equitativas: Las prácticas laborales justas y equitativas deben determinarse en políticas que aseguren el bienestar y protección de sus trabajadores directos y de todas las subcontrataciones que surjan a partir del desarrollo de actividades de la empresa, estas característica incluye aspectos como reclutamiento, selección de personal siguiendo cánones de igualdad, promoción y desarrollo persona, cesación de labores, formación y desarrollo de habilidades, seguridad social Integral, Higienes y seguridad Industrial, asociación colectiva, remuneración.

3. Gestión Ambiental: Las entidades deben ser conscientes de los impactos medio ambientales que generan el desarrollo de su objeto social, por tal razón deben actividades que permitan mitigar esos impactos ambientales y generar contaminación en un grado mínimo. Esta característica incluye aspectos como la Responsabilidad ambiental, el enfoque precautorio, producción más limpia, eco eficiencia, uso de tecnologías sostenibles, gestión de residuos, logística inversa y adquisición sostenible.

4. Practicas Justas de Prestación del Servicio: Es una de las características más fuertes de la RSE, por que se basa en fundamento ético del desarrollo de la actividad empresarial, incluye la realización de alianzas público privadas, la relación con sus filiales, proveedores, clientes, la competencia y las agremiaciones a las cuales por su naturaleza pertenecen. Por ello en este aspecto se deben tener en cuenta la anticorrupción, la competencia justa, la participación política responsable y el respeto a la propiedad privada.

5. Atención Integral de los Usuarios: Esta Característica es vital, sobre todo en el ámbito de la salud, ya que es un servicio público esencial para el desarrollo integral de las personas y el ciudadano. Esta atención integral inicia con el respeto hacia el consumidor, incluye el marketing justo y transparente, la protección de la salud integral de los consumidores, siendo objetivos en la prestación del servicio, suministrando 
elementos farmacéuticos, instalaciones médicas en condiciones que eviten la generación de algún riesgo para el usuario, esto también debe aplicarse para todos los elementos conexo que se requieran para la prestación del servicio. Además de esto las EPS e IPS, deben promover las campañas de prevención y la adquisición de hábitos saludables para promover la Responsabilidad Individual del cuidado de la salud, pero preservando el fácil acceso a los servicios esenciales de Salud, en caso de que se incurra en alguna controversia por alguna falla en el sistema las EPS e IPS deben garantizar un sistema de peticiones, quejas y reclamos; brindando soluciones eficaces que aumenten la satisfacción de los usuarios.

6. Desarrollo Integral de la Comunidad: Este aspecto se refiere a como las EPS e IPS deben brindar desarrollo a las comunidades que se encuentran geográficamente circundantes, este aspecto incluye la participación activa de la comunidad, identificar sus necesidades $\mathrm{u}$ participar activamente en alianza publico privadas que beneficien a la comunidad, promover actividades culturales y apoyar acciones que mejoren la calidad de la educación, el acceso a medios tecnológicos, la empleabilidad y el más importante y pertinente para estas organizaciones la promoción de la Salud, a través de la educación y el suministro de elementos y actividades que mejoren los índices de la comunidad. Pero todas estas actividades se deben dar teniendo como eje transversal la Inversión social.

\section{Materiales y Métodos}

El tipo de método aplicado es el descriptivo aplicado a la muestra de los Estados
Financieros (Balance General y Estado de Resultados) de la ESE hospital Venancio Díaz Díaz para los años 2012-2016. Adicionalmente se aplican indicadores financieros a la muestra para obtener los resultados esperados en el proyecto de investigación. (EVA e indicadores tradicionales) Basado en investigación documental se extrae información relacionada con el cumplimiento de estándares de responsabilidad social empresarial (RSE)

Análisis Financiero Aplicado a los Estados Financieros de la ESE Hospital Venancio Díaz Díaz

En el siguiente aparte se muestra cómo se aplican simultáneamente la metodología tradicional de análisis financiero y la gerencia del valor. Se parte de los indicadores de liquidez, endeudamiento y rentabilidad y el cálculo del valor económico agregado (EVA), aspecto considerado un macro inductor que sirve para evaluar los resultados conforme a la creación de valor (Para la aplicación de los dos sistemas de análisis se parte de los Estados Financieros del hospital adjuntos como primer anexo)

$\mathrm{Se}$ relacionan algunos indicadores tradicionales con su respectiva interpretación:

\section{Tabla 1}

Indicadores de rentabilidad, liquidez y endeudamiento ese hospital Venancio Díaz Díaz.

\begin{tabular}{|c|c|c|c|c|}
\hline Indicadores de liquidez & 2012 & 2013 & 2014 & 2015 \\
\hline Días de cxc & 91.90 & 57.32 & 130.50 & 136.84 \\
\hline Días de proveedores & 235.09 & 72.22 & 170.67 & 216.90 \\
\hline Capital de trabajo & 330.985 & 7.666 & $\begin{array}{c}(851.283 \\
)^{2}\end{array}$ & $\begin{array}{c}(899.938 \\
)^{2}\end{array}$ \\
\hline Prueba acida & $\begin{array}{c}(1.217 .3 \\
41)\end{array}$ & $\begin{array}{c}(2.639 .3 \\
22)\end{array}$ & $\begin{array}{c}(3.596 .2 \\
66)\end{array}$ & $\begin{array}{c}(3.056 .2 \\
11)\end{array}$ \\
\hline $\begin{array}{l}\text { Indicadores } \\
\text { rentabilidad }\end{array}$ & 2012 & 2013 & 2014 & 2015 \\
\hline Rentabilidad activo & $8,85 \%$ & $-2,16 \%$ & $-8,16 \%$ & $-1,37 \%$ \\
\hline Margen bruto & $71,26 \%$ & $28,52 \%$ & $25,17 \%$ & $37,97 \%$ \\
\hline Utilidad neta & $13,57 \%$ & $-1,97 \%$ & $-11,37 \%$ & $-1,78 \%$ \\
\hline $\begin{array}{l}\text { Indicadores } \\
\text { endeudamiento }\end{array}$ & 2012 & 2013 & 2014 & 2015 \\
\hline Endeudamiento total & $12,95 \%$ & $23,79 \%$ & $31,52 \%$ & $28,28 \%$ \\
\hline $\begin{array}{l}\text { Endeudamiento corto } \\
\text { plazo }\end{array}$ & $12,95 \%$ & $23,79 \%$ & $31,52 \%$ & $28,28 \%$ \\
\hline
\end{tabular}


La ESE Venancio Díaz presenta problemas de liquidez reflejados en el deterioro de su capital de trabajo, pasando de ser positivo en los años 2012 y 2013 para disminuir en los años 2014 y 2015, incluso llegando a ser negativo por la alta acumulación de pasivos de corto plazo. Frente al indicador de prueba ácida, se nota absoluta dependencia de la cartera para obtener recursos en el corto plazo. Es claro entonces que la ESE debe comprometer todos sus recursos para cubrir sus pasivos, perdiendo la posibilidad de invertir en infraestructura para mejorar la prestación del servicio.

La anterior situación refleja el problema estructural del sector salud en Colombia: los altos niveles de endeudamiento de los hospitales de la red hospitalaria pública no son resueltos con la rotación esperada de su cartera, lo que conlleva a acumular recursos que no generan la liquidez suficiente para atender los compromisos con proveedores y acreedores en general. Por esta razón durante los años de análisis los pagos promedio a proveedores llegan a niveles alarmantes, de hasta 230 días para cancelar las deudas vencidas.

Frente a la rentabilidad, la ESE genera excedentes únicamente en el primer año, mientras que en los tres siguientes sus costos y gastos superaron los ingresos.

El endeudamiento del hospital puede considerarse sano dado su bajo nivel comparado con su activo. El inconveniente para las finanzas del hospital es la naturaleza del pasivo que está directamente relacionado con su objeto (proveedores) Esta clase de acreedores presenta el riesgo de comprometer el servicio si la deuda no se atiende oportunamente.

Cabe aclarar que el hospital muestra valorizaciones dentro del total de activo, las cuales representan incrementos en el valor de mercado de los terrenos y edificios, pero que en ningún momento pueden ser utilizados en la operación. Por consiguiente, al restarles estos rubros la situación de endeudamiento se relaciona a continuación:
Tabla 2 Indicadores de endeudamiento sin valorizaciones.

\begin{tabular}{lllll} 
Endeudamiento sin valorizaciones & 2012 & 2013 & 2014 & 2015 \\
\hline & $53,79 \%$ & $74,83 \%$ & $95,47 \%$ & $99,93 \%$ \\
\hline Endeudamiento total & $53,79 \%$ & $74,83 \%$ & $95,47 \%$ & $99,93 \%$ \\
\hline Endeudamiento corto plazo & 5
\end{tabular}

Con la disminución de las valorizaciones para el cálculo del indicador, la situación de endeudamiento del hospital es crítica en tanto compromete su capital de trabajo y sus activos fijos para responder a su alto endeudamiento. Las teorías financieras expresan que los activos corrientes deben ser lo suficientemente altos para cumplir con sus obligaciones en el corto plazo, pero ante el deterioro de sus indicadores y el incremento en el nivel de endeudamiento los activos fijos también deben cubrir el monto total de las acreencias.

\section{Aplicación de Macro Inductores de Valor en la ESE Hospital Venancio Díaz}

Mientras los indicadores tradicionales miden la operación de la empresa a partir de informes contables, la gerencia del valor utiliza herramientas que implican el uso de cálculos financieros alineados a las teorías de dirección estratégica y medición del valor de la empresa en marcha (Por ejemplo, el costo de capital). A continuación, se explica la construcción de uno de los macro inductores de valor en la ESE Hospital Venancio Díaz Díaz: Valor económico agregado (EVA), entendido como el remanente que se obtiene al restarle a la rentabilidad de los activos el costo por las fuentes de financiación o costo de capital (Garavito, 2010). 
Tabla 3 Calculo del EVA Operativo Ese Hospital Venancio Díaz.

\begin{tabular}{|c|c|c|c|c|}
\hline & 2012 & 2013 & 2014 & 2015 \\
\hline Activos operación balance general & 2.760 .918 & 4.058 .326 & 4.191 .110 & 3.328 .503 \\
\hline Cxp proveedores & 1.247 .773 & 2.763 .943 & 3.700 .142 & 3.052 .839 \\
\hline Activos netos de operación & 1.513 .145 & 1.294 .383 & 490.968 & 275.664 \\
\hline Utilidad operativa & $(1.067 .730)$ & $(854.816)$ & $(2.664 .296)$ & $(2.581 .142)$ \\
\hline Impuestos & - & - & - & - \\
\hline Utilidad operativa despues de imptos & $(1.067 .730)$ & $(854.816)$ & $(2.664 .296)$ & $(2.581 .142)$ \\
\hline Activos netos de operacion iniciales & 624.459 & 1.513 .145 & 1.294 .383 & 490.968 \\
\hline Costo de capital & $10 \%$ & $10 \%$ & $10 \%$ & $10 \%$ \\
\hline Costo por el uso de los activos & 62.446 & 151.315 & 129.438 & 49.097 \\
\hline Utilidad operacional despues impto & $(1.067 .730)$ & $(854.816)$ & $(2.664 .296)$ & $(2.581 .142)$ \\
\hline Costo por el uso de los activos & $(62.446)$ & $(151.315)$ & (129.438) & $(49.097)$ \\
\hline Ganancia economica o eva operativo & $(1.130 .176)$ & $(1.006 .131)$ & $(2.793 .734)$ & $(2.630 .239)$ \\
\hline Utilidad operativa despues de imptos & $(1.067 .730)$ & $(854.816)$ & $(2.664 .296)$ & $(2.581 .142)$ \\
\hline Activos netos de operacion iniciales & 624.459 & 1.513 .145 & 1.294 .383 & 490.968 \\
\hline Rentabilidad activo neto (ran) & $-170,98 \%$ & $-56,49 \%$ & $-205,84 \%$ & $-525,73 \%$ \\
\hline Costo de capital & $10 \%$ & $10 \%$ & $10 \%$ & $10 \%$ \\
\hline Ganancia economica o eva operativo & $(1.130 .176)$ & $(1.006 .131)$ & $(2.793 .734)$ & $(2.630 .239)$ \\
\hline
\end{tabular}

El cálculo y análisis del EVA se diseña a partir de los activos del balance general, que en el caso analizado se ajustan para restarle las valorizaciones de algunos activos como terrenos y edificaciones, los cuales por no ser parte de la operación del negocio no se incluyen en el total.

Adicionalmente, se toma como costo de capital el 10\%, interés implícito promedio cobrado por los proveedores de bienes o servicios. Tal situación fue descrita anteriormente como reflejo del incumplimiento de las deudas. También puede considerarse como sobre precio en el suministro de dichos bienes o servicios. Solo se considera las deudas con proveedores dada la falta de endeudamiento con el sector financiero.

Se evidencia una clara destrucción de valor para cada uno de los periodos de estudio, partiendo de la pérdida operacional obtenida en cada uno de los años. Si se comparan con los activos de operación, el hospital no obtiene una rentabilidad mínima de los recursos utilizados en su objeto social, por el contrario, la falta de eficiencia organizacional ocasiona graves riesgos en la continuidad del negocio en marcha. (Ver Programa de Saneamiento financiero hospital sesión concejo municipal de sabaneta en anexo 2). Como se puede observar en los estados financiero de la entidad, los ingresos operacionales han experimentados ciclos inestables de aumentos y disminuciones, mientras que sus costos $y$ gastos no presentan un comportamiento acorde a estas tendencias

Otro aspecto considerado como grave en el análisis del EVA del hospital es la composición de los activos netos de operación, valores que presentan una tendencia a la baja por la alta concentración de endeudamiento en proveedores de bienes y servicios, incluso para el año 2015, casi que los activos y pasivos corrientes están en relación 1 a 1 ocasionando graves problemas de liquidez e incumplimiento en el pago de sus obligaciones, entre ellas las deudas con proveedores.

En la siguiente tabla se muestra el cálculo del EVA considerando aspectos no operacionales en su composición: 
Tabla 4 Cálculo del EVA Ese Hospital Venancio Díaz Díaz.

\begin{tabular}{|l|c|c|c|c|}
\hline & 2012 & 2013 & 2014 & 2015 \\
\hline Activos operación balance general & 2.760 .918 & 4.058 .326 & 4.191 .110 & 3.328 .503 \\
\hline Cxp proveedores & 1.247 .773 & 2.763 .943 & 3.700 .142 & 3.052 .839 \\
\hline Activos netos de operación & 1.513 .145 & 1.294 .383 & 490.968 & 275.664 \\
\hline Utilidad despues de impuestos & 1.014 .796 & $(275.617)$ & $(1.035 .528)$ & $(160.607)$ \\
\hline & & & & \\
\hline Activos netos de operacion iniciales & 624.459 & 1.513 .145 & 1.294 .383 & 490.968 \\
\hline Costo de capital & $10 \%$ & $10 \%$ & $10 \%$ & $10 \%$ \\
\hline Costo por el uso de los activos & 62.446 & 151.315 & 129.438 & 49.097 \\
\hline & & & & \\
\hline Utilidad operacional despues impto & 1.014 .796 & $(275.617)$ & $(1.035 .528)$ & $(160.607)$ \\
\hline Costo por el uso de los activos & $(62.446)$ & $(151.315)$ & $(129.438)$ & $(49.097)$ \\
\hline Ganancia economica o eva operativo & 952.350 & $(426.932)$ & $(1.164 .966)$ & $(209.704)$ \\
\hline & & & & \\
\hline Utilidad despues de impuestos & 1.014 .796 & $(275.617)$ & $(1.035 .528)$ & $(160.607)$ \\
\hline Activos netos de operacion iniciales & 624.459 & 1.513 .145 & 1.294 .383 & 490.968 \\
\hline Rentabilidad activo neto (ran) & $162,51 \%$ & $-18,21 \%$ & $-80,00 \%$ & $-32,71 \%$ \\
\hline Costo de capital & $10 \%$ & $10 \%$ & $10 \%$ & $10 \%$ \\
\hline EVA & 952.350 & $(426.932)$ & $(1.164 .966)$ & $(209.704)$ \\
\hline & & & & \\
\hline
\end{tabular}

Comparando la metodología de cálculo del EVA operativo con la determinada en la tabla anterior, se observa como diferencia principal la inclusión de factores no operacionales en su construcción. Ingresos como las transferencias de entes públicos ha mejorado la utilidad neta en algunos años, incluso en el año 2012 el hospital agrega valor por 952.350 millones de pesos, cifra que atenúa la crisis que se evidencia a partir del año 2013. Tal situación se presenta nuevamente en el año 2015, cuando la entidad recibe $\$ 2.043$ millones para gastos de operación, pero la cifra recibida no alcanza a enjugar las pérdidas y vuelve e destruir valor por 209.704 millones.

\section{Análisis de Indicadores de RSE en la ESE Hospital Venancio Díaz Díaz}

Según el informe definitivo de auditoría general de Antioquia para la vigencia 2013 (Contraloría General de la república, 2013), se relacionan a continuación algunos indicadores de RSE
Control de gestión de gestión:

a) Gestión contractual: puntaje 92.77

b) Control financiero: Opinión limpia de los Estados Contables

c) Camas por habitante

Con una capacidad instalada de 37 camas, la ESE Hospital logra cubrir las necesidades básicas en salud de aproximadamente 48.266 habitantes, lo cual significa que se posee 1 cama por cada 1.304 habitantes.

Médicos por habitante.

La ESE Hospital presenta 4 médicos para dar cubrimiento en salud a los 48.266 habitantes de la localidad, o sea que existe un médico por cada 12.066,50 habitantes.

Enfermeras por habitante.

La ESE Hospital presenta 11 enfermeras para dar cubrimiento en salud a los 48.266 habitantes de la localidad, o sea que existe una enfermera por cada $4.387,82$ habitantes.

Gobernanza e Inversión Transparente. 
Controles de enfermería realizados.

Por cada 100 habitantes que posee el municipio, la ESE Hospital realiza 956 controles de enfermería; es decir al 0,02 de los habitantes que posee el municipio se les realiza controles de enfermería.

Atenciones médicas electivas realizadas

Durante la vigencia el Hospital realizó 9.717 consultas médicas electivas (general especializada); que corresponden 0,20 consultas por habitantes.

Atención odontológica

La ESE Hospital realizó 3.483 consultas odontológicas; En promedio corresponde a 0,07 consultas odontológicas por habitante del municipio.

\section{Conclusiones}

La aplicación del macro inductor del EVA en los Estados Financieros de la ESE Hospital Venancio Díaz Díaz muestra un claro y continuo deterioro en la generación de valor, ocasionado principalmente por la ineficiencia operativa reflejada en costos de funcionamiento que superan los ingresos de operación.

Adicionalmente, el endeudamiento con proveedores de servicios ha afectado la composición de los activos de operación generando graves consecuencias en el suministro de bienes y servicios

Según la muestra extraída para valorar la RSE empresarial de la ESE Hospital Venancio Díaz, se observa un cubrimiento medio de las necesidades en salud del municipio de Sabaneta. Indicadores como cama por habitante, doctores y enfermeras por habitante reflejan un nivel medio de cobertura en la operación de la entidad

Los graves problemas financieros expuestos en la evaluación del EVA de la entidad muestran indiscutiblemente que la cobertura en las prácticas de RSE sacrifican los recursos dispuestos para la operación del hospital.
Lo anterior es concluyente si se analiza que la entidad compromete seriamente su continuidad tratando de responder a una sociedad que requiere servicios de salud de calidad.

Como consecuencia de los problemas estructurales del sector salud en Colombia, la ESE Hospital Venancio Díaz no puede cumplir con los dos propósitos objeto de este proyecto de investigación: rentabilidad y prácticas de RSE. La anterior conclusión se evidencia en el continuo deterioro del EVA como indicador de cumplimiento del principio de negocio en marcha.

\section{Referencias}

Álvarez, A. G. (2004). Introducción al Análisis Financiero Tercera Edición. Alicante: Editorial Clud Universitario.

Andrade, S., \& Fernández, M. (2010). Responsabilidad social empresarial: significados e implicaciones a partir de la evolución de los procesos productivos. Producción económica, 23.

Barcelona, D. U. (2003). La dirección y el control estratégico. Su aplicación en los Recursos Humanos. Obtenido de http://diposit.ub.edu/dspace/bitstream/24 45/13220/3/Fundamentos\%20(Parte\%20 primera).pdf

Burgos, B. M., Montes, L. S., \& García, M. C. (2013). Análisis Financiero y gerencia del valor de las empresas sociales del Estado. Consideraciones a partir del estudio a la ESE Hospital Universitario Erasmo Meoz. Cúcuta: Universidad Francisco de Paula Santander.

Colina, M. V. (2016). Gerencia Basada en Valor: La Inclusión del Costo Financiero como un Costo de Oportunidad. Actualidad Contable FACES Año $9 \mathrm{~N}^{\mathrm{o}} 13$, JulioDiciembre 2006, 154-165.

Congreso de la República. (1993). Ley 100 de 1993. Artículo 197. Numeral 2. 
Congreso de la República. (1993). Ley 100. Artículo 194.

Contraloría departamental de Antioquia. (Septiembre de 2015). Informe auditoría integral ESE Hospital Venancio Diaz Vigencia 2014. Obtenido de http://www.cga.gov.co/ProcesosMisional es/Auditoriaintegral/Informes $\% 20$ Fiscale s\%20y\%20Financieros\%202015/ESE\%2 OSabaneta.pdf

Contraloría General de la república. (Diciembre de 2013). Auditoría Integral Hosptal Venancio Diaz vigencia 2013. Obtenido de

http://www.cga.gov.co/ProcesosMisional es/Auditoriaintegral/Informes $\% 20 \mathrm{de} \% 20$ Auditora\%202014/ESE\%20Hospital\%20 Venencia\%20Diaz\%20Diaz\%202014.pdf

Garavito, J. C. (2010). ¿Crean o destruyen valor las empresas del sector solidario en Colombia? Caso de los fondos de empleados. Revista MBA EAFIT, 48-55.

García, O. L. (2003). Valoración de empresas, gerencia del valor y EVA. Bogotá: ECOE ediciones.

Mares, A. I. (Octubre de 2009). Desarrollo del análisis factorial multivariable aplicado al análisis financiero actual. Obtenido de http://www.eumed.net/librosgratis/2010a/666/METODOLOGIA $\% 20$ DEL\%20ANSLISIS\%20FINANCIERO \%20TRADICIONAL.htm

Méndez, C. A. (2007). EBITDA, ¿Es un indicador financiero contable? CAPIV REVIEW Vol. 5, Pagina 41- 50.

Minsalud. (Agosto de 1994). Decreto 1876 de 1994. Obtenido de http://www.hospitalpaipa.gov.co/wp-
content/uploads/2013/03/Decreto-1876de-1994.pdf

Morales, J., \& Galeano, C. (2013). Enfoque de la Responsabilidad Social Empresarial (RSE) en las EPS del régimen. Manizales: Universidad de Manizales.

Moreno, S. G. (Diciembre de 2006). El control. Obtenido de http://www.economicas.unsa.edu.ar/web/ archivo/otros/control-clase-15-10-10.pdf

Rosillón, M. A. (Diciembre de 2009). Revista Venezolana de Gerencia Vol 14. Obtenido de

http://www.scielo.org.ve/scielo.php?scrip $\mathrm{t}=\mathrm{sci}$ arttext\&pid=S1315$\underline{99842009000400009}$

Triviño, A. F. (2013). Coyuntura del sistema de salud en Colombia: Caracterización de una crisis desde las particularidades financieras de las EPS. Revista Gestión y desarrollo , 103-116.

Universal, P. E. (2016). Uno de cada cuatro hospitales en el país están en alto riesgo financiero. Cartagena: Periódico el Universal.

Vera Colina, M. (Julio-Diciembre de 2006). Gerencia Basada en Valor: La Inclusión del Costo Financiero como un Costo de oportunidad. Actualidad Contable FACES Año 9 No 13, 154-165.

Vergara, R. E. (Octubre de 2013). Aplicación del control fiscal en Colombia para las empresas públicas y privadas que administran recursos del estado. Obtenido de

http://repository.unimilitar.edu.co/bitstrea $\underline{\mathrm{m} / 10654 / 11491 / 2 / \mathrm{ENSAYO} \% 20 \mathrm{CONTR}}$ OL\%20FISCAL\%20RECV.pdf 